Case Report

\title{
A case series of nontyphoidal Salmonella bacteraemia-some food for thought!
}

\author{
FN Mubarak ${ }^{1}$, P Chandrasiri $^{1}$ \\ Sri Lankan Journal of Infectious Diseases 2013 Vol.3(2):37-42
}

DOI: http://dx.doi.org/10.4038/sljid.v3i2.4711

Key words: Nontyphoidal Salmonella; bacteraemia; compromised patients

\begin{abstract}
Nontyphoidal salmonellae (NTS) are important foodborne pathogens. Mild to moderate gastroenteritis in healthy young adults is often self-limiting and requires no antibiotic treatment. Compromised hosts can develop severe disease complicated by bacteraemia and focal infection which may lead to devastating consequences. Bacteraemia, especially if it occurs without gastrointestinal symptoms should prompt careful clinical and microbiological evaluation. Appropriate antibiotics given in the correct doses for the recommended duration is vital for satisfactory outcome to completely eliminate the infection and prevent recurrences. Medical therapy should be combined with surgical management when indicated. Rational and controlled antibiotic use in human, veterinary and agriculture practices is essential to counter the increasing resistance that is developing in Salmonella worldwide. Adherence to good food hygiene practices will help prevent infections. We describe a case series of NTS bacteraemia in patients with underlying compromised conditions detected over a period of six months in the Microbiology Department of the National Hospital of Sri Lanka.

\section{Introduction}

Nontyphoidal Salmonella (NTS) are important foodborne pathogens. Young adults with mild to moderate gastroenteritis should be managed without antibiotics. Severe disease may occur at the extremes of age. Conditions that affect the body's defense system against enteric and intracellular pathogens such as gastric hypoacidity, alteration of bowel flora, diabetes, malignancy, rheumatological disease, therapeutic immunosuppression, HIV infection and reticuloendothelial disease (malaria, sickle cell disease) predispose to invasive infection. Anatomical anomalies (kidney stones, gallstones, atherosclerotic lesions, prosthetic devices) may serve as foci of infection. ${ }^{1}$
\end{abstract}

\section{Case Series}

${ }^{1}$ National Hospital of Sri Lanka, Colombo 10, Sri Lanka

Address for correspondence: Dr N Mubarak, 68A, Davidson Road, Colombo 4, Sri Lanka

Email: nsmya@yahoo.com Tele No: +94(0)777 518338 
During the period of 6 months between $21^{\text {st }}$ February and $20^{\text {th }}$ August 2012, four isolates of NTS were detected in blood cultures at the Microbiology Laboratory of the National Hospital of Sri Lanka (NHSL). Further identification of the isolates was done at the Medical Research Institute, Colombo, Sri Lanka which is the National Reference Laboratory and PathWest Laboratory Medicine, Western Australia.

\section{Case 1}

An 80 year old male with no known co-morbidities was admitted to the local hospital complaining of loss of appetite, loss of weight and bilateral limb swelling of a few weeks duration. Ultra sound scan of the abdomen revealed multiple liver lesions and he was transferred to the NHSL for CT scan abdomen and further investigations. He was diagnosed to have hepatocellular carcinoma with background cirrhosis. The patient developed fever during his stay and blood culture yielded Salmonella serotype Enteritidis after 24 hours incubation. He was treated with intravenous cefotaxime $1 \mathrm{~g}$ 8hourly and transferred back to the local hospital on the $4^{\text {th }}$ day of antibiotic treatment following clinical improvement. He was subsequently discharged on the $7^{\text {th }}$ day of treatment.

\section{Case 2}

A 65 year old female with a past history of diabetes, hypertension and chronic liver parenchymal disease was admitted following a two day history of vomiting, confusion and abdominal distention. She was afebrile on admission but developed fever subsequently. Blood culture taken on admission grew Salmonella serotype Corvallis after 24 hours incubation. She was treated with intravenous ceftriaxone $2 \mathrm{~g}$ daily for 5 days and discharged after clinical improvement on oral ciprofloxacin 500mg 12 hourly and oral metronidazole 400mg 8hourly for 5 days.

\section{Case 3}

A 40 year old female with no known co-morbidities was admitted for a planned ureteroscopy and laserlithotripsy. During the procedure a biopsy was taken from the bladder. On the day following the procedure, she developed fever with vomiting, abdominal pain and haematuria. Blood cultures were taken before starting intravenous ceftazidime 1g 8hourly. Salmonella spp. group B $4(5) ; b ; 1,2$ was isolated after 48 hours incubation. As she was running a high fever, the antibiotic was changed to intravenous meropenem $1 \mathrm{~g}$ 8hourly, following culture positivity. Subsequently the isolate was shown to be sensitive to ciprofloxacin and ceftriaxone. However meropenem was continued for 5 days. She was discharged on the $5^{\text {th }}$ post-operative day following clinical improvement.

\section{Case 4}

A 66 year old female, diagnosed with chronic myeloid leukemia in 2011 and having no other comorbidities was admitted with a complaint of fever for 2 days, accompanied by shortness of breath and mild central chest pain. She had been given radiotherapy and chemotherapy and the follow up had been uneventful. Intravenous cefotaxime $1 \mathrm{~g}$ 8hourly was started after taking blood 
cultures which yielded Salmonella serotype Enteritidis after 24 hours. As the fever was persistently high, the antibiotic was changed to intravenous meropenem $1 \mathrm{~g}$ 8hourly on day 3 and continued for 21 days despite the isolate being sensitive to ceftriaxone. 2D echo and ultrasound scan abdomen showed no significant findings. She was discharged upon clinical improvement.

\section{Discussion}

Approximately 5\% of cases of NTS gastroenteritis are complicated by bacteraemia. It is a serious and potentially fatal problem and mortality rates of $16 \%$ and $18 \%$ have been reported in studies from Spain and USA respectively. ${ }^{1}$ If bacteremia with NTS occurs without gastrointestinal symptoms, it is an ominous sign and should alert the clinician to investigate for an underlying immunosuppressive illness or the presence of an anatomical risk factor. Immunocompromised hosts are more likely to develop bacteremia and focal infections such as infectious endarteritis, endocarditis, arthritis, osteomyelitis, meningitis, pneumonia and abscess formation in the liver and spleen. A feared complication in infants is the development of CNS infections. Relapses are a common problem due to inadequate elimination of infective foci. ${ }^{1}$

Uncomplicated bacteraemia can be treated with 10-14 days of antimicrobial therapy. If cultures remain positive or there is no clinical improvement it is essential to investigate further with echocardiography, CT scan or angiogram to look for foci of infection. ${ }^{1}$

A potentially fatal complication that occurs in older adults is the development of infectious endarteritis, especially in the abdominal aorta. Bacteria settle in the atherosclerotic plaques and serve as a nidus of infection. The overall survival rate is approximately $62 \%$ with combined medical and surgical therapy. ${ }^{1}$

Salmonella endocarditis has an aggressive and destructive course, often requiring valve replacement. Following successful surgery, a minimum of 6 weeks antibiotic therapy followed by months of suppressive therapy is needed for a satisfactory outcome. If residual infection cannot be ruled out, years if not lifelong suppressive therapy may be needed. ${ }^{1}$

Foci of infection can develop at any anatomical site. These infections often become problematic as they may persist despite clearance of bacteraemia. Soft tissue infection should be drained or debrided whenever possible, followed by a minimum of 2 weeks of antibiotics. However 4-6 weeks of therapy is advisable. The most problematic infections occur in patients with sickle cell anaemia who develop arthritis and osteomyelitis. Splenectomy is often needed for splenic abscesses. Infections in a structurally and functionally abnormal urinary tract require prolonged courses of antibiotics that are excreted in the urine to eradicate the infection. ${ }^{1}$

NTS infections and bacteraemia are commonly associated with HIV infection in the developing world. It is also used as a disease marker for this illness. ${ }^{2}$ Reports from Africa have shown that despite Salmonella enterica serotype Typhi and Salmonella enteric serotype Paratyphi being endemic in these areas, NTS bacteraemia is the second most frequent blood isolate (following mycobacteria) in 35\% of HIV infected adult patients. Prolonged therapy of 4-6 weeks is thought to be needed to eradicate infection and prevent relapses. ${ }^{1,2}$ 
NTS were initially sensitive to ampicillin, chloramphenicol and co-trimoxazole. A multidrug resistant strain (DT104) of Salmonella enteric serotype Typhimurium emerged in the 1990's and spread worldwide. It was resistant to the above mentioned antibiotics and to streptomycin and tetracycline (resistant type ACSSuT). Subsequent treatment options were flouroquinolones and $3^{\text {rd }}$ generation cephalosporins. ${ }^{1}$ Antibiotic use in agriculture and veterinary practices, not only for disease treatment and prevention but also as growth promoters, led to the emergence of Salmonella strains with reduced susceptibility to flouroquinolones in Europe and the USA., Antibiotics are also being used and misused in Sri Lanka in agriculture and veterinary practices. The contribution of this to the rising rate of antimicrobial resistance is unknown. ${ }^{4}$ Salmonella strains resistant to extended spectrum cephalosporins have been reported from the USA and Asia. ${ }^{1,3,5}$ It has also been shown that development of bacteremia is linked to infection with resistant strains. 6,7

Due to the emergence of resistance, empirical therapy for life threatening NTS infections should consist of a $3^{\text {rd }}$ generation cephalosporin and a fluoroquinolone followed by de-escalation to a single bactericidal drug such as ampicillin, $3^{\text {rd }}$ generation cephalosporin or a fluoroquinolone based on susceptibilities. There is no consensus over whether a flouroquinolone or a $3^{\text {rd }}$ generation cephalosporin is the better choice, except for CNS infections where high dose ceftriaxone is the best choice for optimal penetration of the blood brain barrier. ${ }^{1}$ In this case series, treatment was not given for the recommended duration for uncomplicated bacteraemia in cases 1 to 3 . Despite all four NTS isolates being sensitive to ciprofloxacin and ceftriaxone, we noted a reluctance to de-escalate in the management of cases 3 and 4.

Inappropriate usage of antibiotics by doctors, other health personnel and patients is driving the development of resistance. Fluoroquinolone resistance rates among Salmonella enteric serotype Typhi and Salmonella enteric serotype Paratyphi is increasing and has approached almost 100\% in Salmonella enterica serotype Paratyphi in Sri Lanka. ${ }^{8}$ There is a pressing need to create awareness and promote the rational use of antibiotics, together with controlling the availability of over the counter drugs. Suitable alternatives to antibiotics as growth promoters and the development of policies for antibiotic use in veterinary and agriculture practices should be encouraged.

Approximately 95\% of cases of Salmonella infection are foodborne. Many food sources, such as eggs, poultry, cheese, dry cereal, ice cream premix, juices and fresh vegetables have been implicated in outbreaks. Eggs and poultry are common food sources. Faecal contamination of cracked egg shells and transovarian infection of grade A eggs with intact shells lead to infection. Fully cooked or boiled eggs or the use of pasteurized eggs can prevent infection. Studies done in the USA have found that on average 1 in 8 of sampled broiler chickens were contaminated with Salmonella. This leads to contamination of other food items when common cutting boards and utensils are used during food preparation. Food borne disease in the community, especially in high risk individuals, can be reduced through education on food preparation practices and ensuring that poultry dishes are thoroughly cooked by ensuring a minimal internal temperature of $74^{\circ} \mathrm{C}\left(165^{\circ} \mathrm{F}\right) .^{9}$ 


\section{Conclusion}

NTS bacteraemia is a serious complication, especially at the extremes of age and in people with underlying co-morbidities or immunosuppression. This case series highlights its seemingly common occurrence in this group of patients. With the increase in patronage of restaurants and food outlets, such cases are likely to be seen more frequently. Immunocompromised patients and their caregivers need to be educated regarding good food hygiene practices. Appropriate antibiotics given for the recommended duration is vital for a satisfactory outcome in Salmonella bacteraemia. Rational antibiotic use in medical practice, regulating the availability of over the counter drugs, together with responsible and controlled antibiotic use in agriculture and veterinary practice is a must to prevent increased morbidity and mortality due to the development of antimicrobial resistance.

Conflicts of Interest : No conflicts of interest.

\section{Acknowledgements :}

We acknowledge the assistance of Dr Sujatha Pathirage Medical Research Institute, Colombo 8, Sri Lanka and Dr Tim Inglis of PathWest Laboratory Medicine, Western Australia in the identification of the isolates.

\section{References}

1. Hohmann EL. Nontyphoidal salmonellosis. Clin Infect Dis 2001; 32(2):263-269. http://dx.doi.org/10.1086/318457

2. Morpeth SC, Ramadhani HO \& Crump JA. Invasive non-typhi Salmonella disease in Africa. Clin Infect Dis 2009; 49:606-611 doi:10.1086/603553

3. $\mathrm{Su} \mathrm{LH}$, Chiu $\mathrm{CH}$, Chu $\mathrm{C} \& \mathrm{Ou}$ JT. Antimicrobial resistance in nontyphoidal salmonella serotypes: A global challenge. Clin Infect Dis 2004; 39: 546-551 doi:10.1086/422726

4. Corea E. Antibiotic use in animal husbandry and its effects on human health. The Bulletin of the Sri Lanka College of Microbiologists 2011; 9(1): 53-5. No doi

5. Jabeen K, Zafar A, Irfan S, Khan E, Mehraj V \& Hasan R. Increase in isolation of extended spectrum beta lactamase producing multidrug resistant nontyphoidal salmonellae in Pakistan. BMC Infect Dis 2012;10:101. doi:10.1186/1471-2334-10-101

6. Gordon MA, Graham SM, Walsh AL, Wilson L, Phiri A, Molyneux E et al. Epidemics of invasive Salmonella enterica Serovar Enteritidis and $S$ enterica Serovar Typhimurium infection associated with multidrug resistance among adults and children in Malawi. Clinical Infectious Diseases 2008; 46:963-9. doi:10.1086/529146

7. Varma JK, Melbak K, Barret TJ, Beebe JL, Jones TF, Rabatsky-Her T et al. Antimicrobial-resistant nontyphoidal Salmonella is associated with excess bloodstream infections and hospitalizations. J Infect Dis 2005; 191(4):554-561. http://dx.doi.org/10.1086/427263

8. Chandrasiri NS, Jayawardhana JMDD, Ratnayake NR, Feroza MBF, Cooray KJ, Menike RMW et al. Changing pattern of bacteraemic salmonella serotypes and antibiotic sensitivity (ABST) in a tertiary care hospital from 2006-2009. The Bulletin of the Sri Lanka College of Microbiologists 2010; 8(1): 17-18. No doi 
9. Salmonella serotype Enteritidis - General information. Centers for Disease Control and Prevention. http://www.cdc.gov/nczved/divisions/dfbmd/diseases/salmonella_enteritidis/ 